\title{
THE JOURNAL OF PHYSICAL CHEMISTRY
}

\section{C: Surfaces, Interfaces, Porous Materials, and Catalysis}

Subscriber access provided by lowa State University | Library

\section{Moving Behavior of Nano-Droplets on Wedge-Shaped Functional Surfaces}

Shuai Wang, Chao Wang, Zhilong Peng, and Shaohua Chen

J. Phys. Chem. C, Just Accepted Manuscript • DOI: 10.1021/acs.jpcc.8b09831 • Publication Date (Web): 03 Jan 2019

Downloaded from http://pubs.acs.org on January 7, 2019

\section{Just Accepted}

"Just Accepted" manuscripts have been peer-reviewed and accepted for publication. They are posted online prior to technical editing, formatting for publication and author proofing. The American Chemical Society provides "Just Accepted" as a service to the research community to expedite the dissemination of scientific material as soon as possible after acceptance. "Just Accepted" manuscripts appear in full in PDF format accompanied by an HTML abstract. "Just Accepted" manuscripts have been fully peer reviewed, but should not be considered the official version of record. They are citable by the Digital Object Identifier (DOI@). "Just Accepted" is an optional service offered to authors. Therefore, the "Just Accepted" Web site may not include all articles that will be published in the journal. After a manuscript is technically edited and formatted, it will be removed from the "Just Accepted" Web site and published as an ASAP article. Note that technical editing may introduce minor changes to the manuscript text and/or graphics which could affect content, and all legal disclaimers and ethical guidelines that apply to the journal pertain. ACS cannot be held responsible for errors or consequences arising from the use of information contained in these "Just Accepted" manuscripts. 


\title{
Moving Behavior of Nano-Droplets on Wedge-Shaped Functional Surfaces
}

\author{
Shuai Wang a, c Chao Wang b, 1 Zhilong Peng a, c Shaohua Chen a, c, 2 \\ a Institute of Advanced Structure Technology, Beijing Institute of Technology, Beijing, 100081, China \\ ${ }^{\mathrm{b}}$ LNM, Institute of Mechanics, Chinese Academy of Sciences, Beijing, 100190, China \\ ${ }^{\mathrm{c}}$ Beijing Key Laboratory of Lightweight Multi-functional Composite Materials and Structures, Beijing \\ Institute of Technology, Beijing, 100081, China
}

\begin{abstract}
:
Surface functionalization has important application prospect in many aspects, for example, anti-drag, anti-pollution, anti-icing, self-cleaning, reversible adhesion, water collection, etc. Recently, a new technique of functional surface was proposed, in which a droplet can be activated to move from the narrow end of a wedge-shaped hydrophilic region imbedded in a hydrophobic surface to the wide end. Systematic molecular dynamic simulations and theoretical analysis are carried out in this paper in order to understand the motion mechanism and more systematic designs for advanced functional surfaces are further proposed. It is found that the potential energy of droplet decreases continuously as a function of distance when the droplet moves from the narrow end of the wedge-shaped track to the wide end and the corresponding potential energy achieves the minimum when the droplet completely enters the hydrophilic region. Effects of the wedge angle of hydrophilic area and the size of droplet on the moving behavior are systematically investigated. It is interesting to find that both factors would
\end{abstract}

\footnotetext{
${ }^{1}$ Corresponding author. E-mail: wangchao@lnm.imech.ac.cn
} ${ }^{2}$ Corresponding author. E-mail: chenshaohua72@hotmail.com 
show significantly influence on the moving behavior of droplets, which is well consistent with the experimental observations. Theoretical analysis is further carried out, where driving force of droplet transport is well achieved. All the MD simulation results and experimental observations can be well explained by the nonlinearly changed driving force. Finally, several new designs of functional surfaces for controlling droplet transport, including droplet turning, pinning, accelerating, gathering and moving path selection are suggested, the feasibilities of which are further verified and discussed. The results in this paper would be helpful for the design of advanced functional surfaces to manipulate droplets in real applications. 


\section{INTRODUCTION}

Besides the transport of solid particles, ${ }^{1-5}$ motion of liquid droplets on a solid surface has achieved wide interests in both scientific and industrial fields due to its vital role in many fields, like self-cleaning textiles fabrication, ${ }^{6-7}$ corrosion inhibition, ${ }^{8}$ anti-freezing, ${ }^{9}$ water collection, ${ }^{10-}$ ${ }^{11}$ liquid transportation, ${ }^{12-13}$ droplet-based micro-fluidics ${ }^{14}$ and oil-water separation, ${ }^{15}$ etc. Lu et al. ${ }^{16}$ gave a good summary on recent research on dynamic wetting from newtonian fluids to nonnewtonian fluids and nanofluids while others focus on solid surfaces. The wettability of solid surfaces is the main factor of concern, which can be modified fortunately and effectively by changing the surface chemical composition ${ }^{17}$ and/or microstructures. ${ }^{18-19}$ For example, the hydrophobicity of surfaces with pillar and groove micro-structures can be enhanced by increasing the pillar height or roughness and becomes constant beyond certain values of pillar height. ${ }^{18}$ It can also be tuned dynamically by varying the external fields, for example, electrical potential, ${ }^{20}$ ultraviolet irradiation, ${ }^{21}$ extension ${ }^{22}$ and temperature. ${ }^{23}$ Based on the wettability gradient of surfaces, several approaches regarding the drive of droplet motion have been reported. ${ }^{12,}{ }^{24-26}$ Zhang et al. ${ }^{27}$ proposed another interesting concept of shape gradient, i.e., a wedge-shaped or triangular-shaped hydrophilic surface embedded in a hydrophobic surface to obtain a composite surface (called as a wedge-shaped or triangular-shaped functional surface), to drive water droplet self-running. Hkoo and Tseng fabricated a wedge-shaped chemically patterned gradient surface to obtain the spontaneous motion of water droplets for a wide range of water droplet volume, in which the highest reported velocity of water droplets on such a gradient surface is $0.5 \mathrm{~m} / \mathrm{s}$ so far..$^{28}$ Alheshibri et al. ${ }^{29}$ made a hydrophilic aluminum wedge surface based on a hydrophobic copper and found that water droplets move towards the end of 
the wedge and contact more aluminum surface area. They found that the mode of droplet motion depends on the wedge angle and the moving speed depends on both the wedge angle and contact angles on $\mathrm{Al}$ and $\mathrm{Cu}$ surfaces. Zheng et al. ${ }^{30}$ made a wedge-shaped super-hydrophobic copper surface with a polydimethylsiloxane (PDMS) oil layer to achieve droplet motion, in which the wedge angle was also found to be a key factor influencing the maximum moving velocity and position of droplets. Ghosh et al. ${ }^{31}$ and Ody et al..$^{32}$ adopted the similar shape-gradient scheme to study more complex manipulations, including liquid metering, merging, dispensing, splitting and stop of an individual ferrofluid droplet with a uniform magnetic field.

Although the wedge-shaped functional surface has been experimentally proved to be a feasible way for actuation of droplet motion and some simple functions have been achieved, it is not enough to meet the requirement of many real applications, like lab-on-a-chip ${ }^{14,33}$ to study aggregation and mixing of droplets required for the kinetics of biological and chemical reactions. Controlling path would be much helpful for the separation and classification of droplets containing different substances. Rapid and precise control of droplets should also be useful in some real applications, which may be realized by the pinning method. Therefore, more efforts are still required to find potential skills for more real functions, such as controlling the moving direction of droplets, accelerating, pinning or stopping a moving droplet, gathering discrete droplets, etc.

In this paper, both the molecular dynamic simulation method (MD) and theoretical model are adopted to study the mechanical mechanism of droplet motion on wedge-shaped functional surfaces. The main influencing factors of droplet transport, such as the wedge angle and size of the droplet are discussed, the results of which are compared with the existing experimental 


\section{SIMULATION DETAILS}

\subsection{Numerical model}

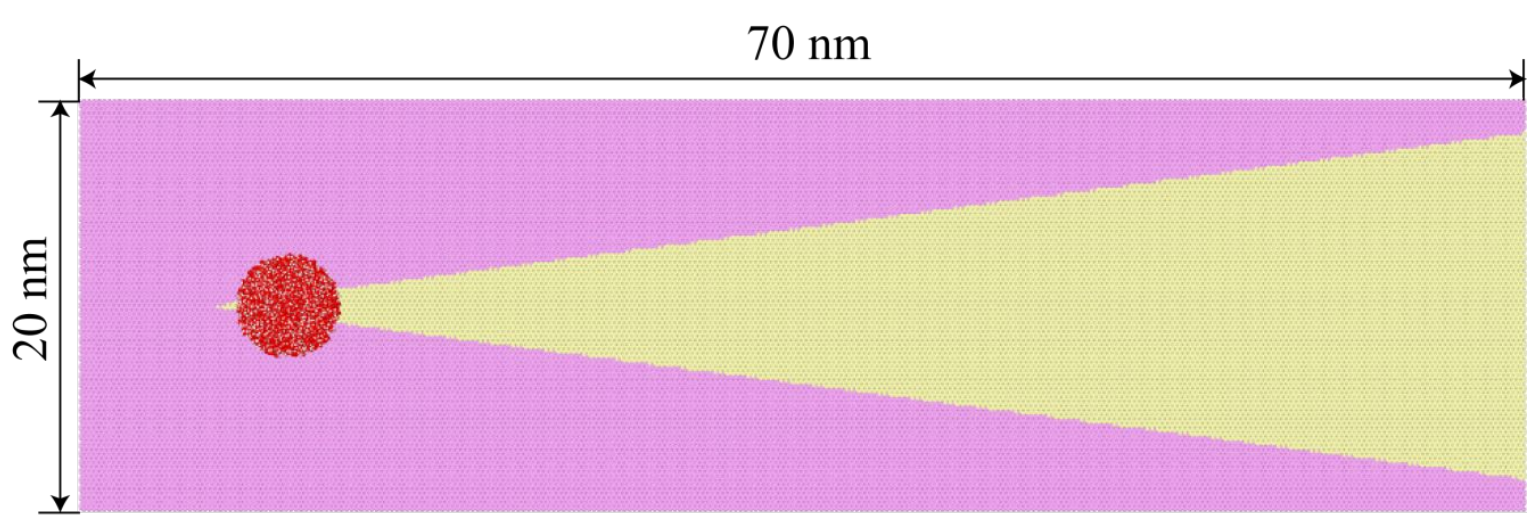

(a) top view

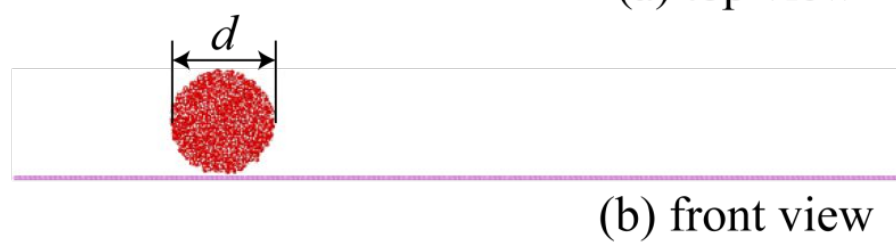

Figure 1. Schematic of the simulation system. (a) the top view; (b) the front view.

A wedge-shaped hydrophilic zone is embedded in a $20 \mathrm{~nm}$-wide and $70 \mathrm{~nm}$-long rectangular hydrophobic substrate surface, where the wedge angle is $15^{\circ}$. Without loss of generality, we use graphene as the substrate. A spherical water droplet of diameter $d$ is put at the wedge end as shown in Figure 1a. In our simulation, the water droplet is treated as a standard model (SPC/E) $)^{34-}$ ${ }^{35}$ with the SHAKE algorithm. ${ }^{36}$ Besides the SPC/E model, TIP3P ${ }^{37}$ and TIP4P ${ }^{38}$ models have also been considered, the spontaneous motion simulated by three models are almost the same, the corresponding analysis can be found in Figure S1 of Supporting Information. The long-range Coulomb interaction in water droplet is computed by particle-particle particle-mesh (PPPM) 
algorithm. ${ }^{39}$ The C-C interaction in the graphene substrate with different wettability is described through AIREBO potential. ${ }^{40}$ Interaction between the oxygen atom in water droplet and carbon atom in graphene is described by Lennard-Jones potential $\varphi(r)=4 \varepsilon\left[(\sigma / r)^{12}-(\sigma / r)^{6}\right]$, in which the equilibrium distance $\sigma$ takes $0.319 \mathrm{~nm}$. However, the well depth $\varepsilon$ would take two values of 5.85 and $1.95 \mathrm{meV}$, which correspond to $51^{\circ}$ and $138^{\circ}$ contact angles, respectively ${ }^{41-42}$ and could be achieved through charge-carrier density ${ }^{43}$ or oxygen-containing functional groups ${ }^{44}$ experimentally. The temperature of water droplet remains $300 \mathrm{~K}$ with the Nosé-Hoover thermostat. The cutoff distance is set as $1 \mathrm{~nm}$ and time step is $1 \mathrm{fs}$ in all simulations. All simulations are implemented with an open source software Large-scale Atomic/Molecular Massively Parallel Simulator (LAMMPS). ${ }^{45}$

\subsection{Numerical analysis}
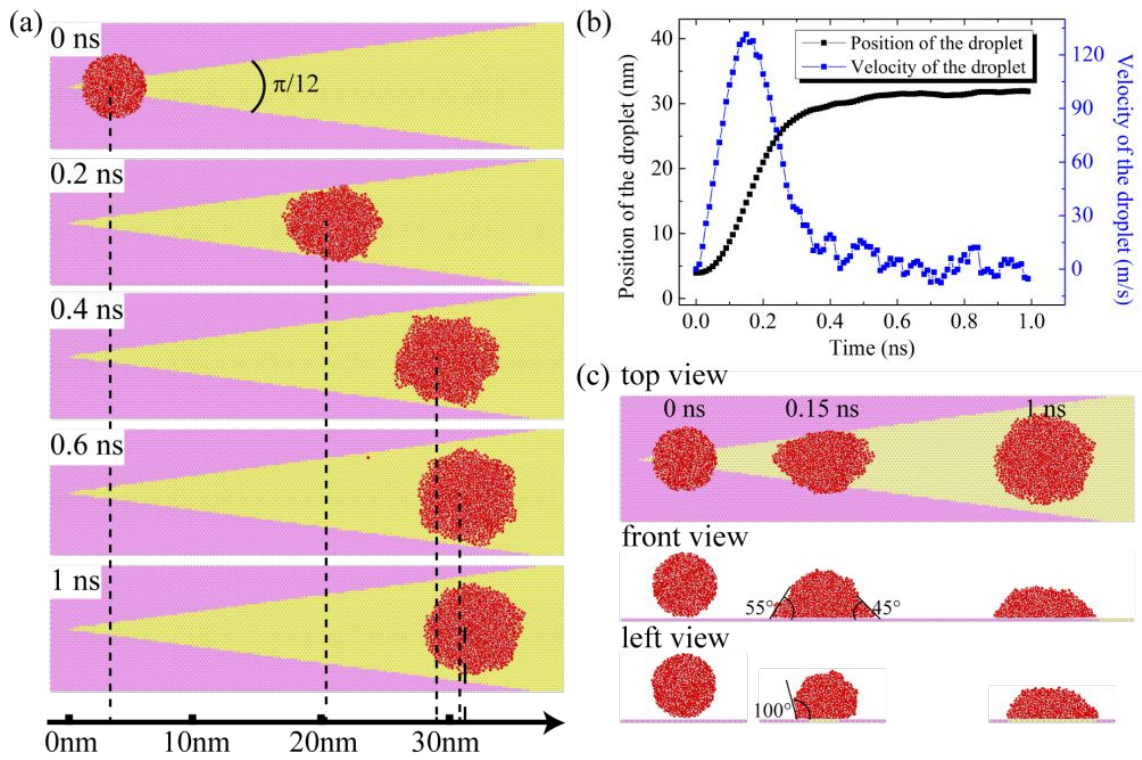

(c) top view

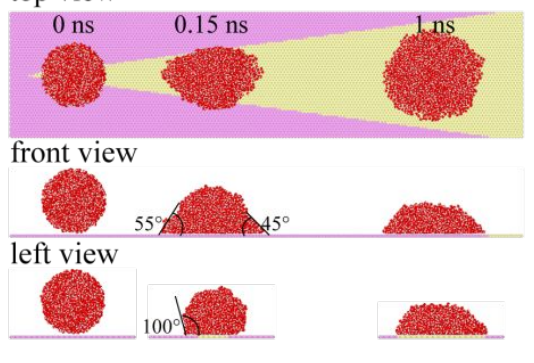

Figure 2. A water droplet moves spontaneously within an embedded wedge-shaped region on a graphene sheet. (a) Snapshots of the position of droplet on the wedge-shaped functional surface at 0, 0.2, 0.4, 0.6 and $1 \mathrm{~ns}$; (b) Position and velocity of the water droplet as a function of the collapsed time; (c) Snapshots of the configuration of a moving droplet observed in different directions. The diameter of the droplet $d$ is $5 \mathrm{~nm}$. 
In order to achieve the energy minimization, the droplet is initially placed far away from the interface of the hydrophobic and hydrophilic areas, after which the droplet is kept at $300 \mathrm{~K}$ with Nosé-Hoover thermostats for a long time to reach thermal equilibrium and optimal configuration. Then, the droplet is put at the narrow end of the wedge-shaped hydrophilic region to study its transport mechanism as shown in Figure 2a. Once the droplet is released, it moves spontaneously to the right tail in the wedge-shaped hydrophilic region. Finally, it stops after completely entering the hydrophilic region without contact between the boundary of water droplets and the boundary between hydrophobic and hydrophilic areas. Snapshots at $0,0.2,0.4$, 0.6 and 1ns are shown in Figure 2a and a typical movie S1 is given in Supporting Information. The instant position and velocity of the droplet are recorded in Figure $2 b$. From Figure $2 b$, it shows that the distance from the initial wedge end increases very quickly at the early stage and then increases slowly at the consequent stage. Finally, the droplet will oscillate at a certain place. While the instant moving velocity of the droplet increases sharply at the initial stage and achieves the maximum before the moment of the whole droplet entering the hydrophilic area.

The varying trend of velocity can be easily explained by the net force acted on the droplet as shown in Figure S2 of Supporting Information, which can be directly achieved in MD simulation. Initially, the net force is positive, which leads to a positive acceleration and an increasing velocity of droplets. When the net force vanishes at about $0.15 \mathrm{~ns}$, the velocity of droplets reaches to the maximum. At this moment, the droplet is about $20.7 \mathrm{~nm}$ far away from the narrow end of the wedge-shaped hydrophilic region as shown in Figure $2 \mathrm{~b}$ and the corresponding configuration is shown in Figure 2c. After that, the net force becomes negative, which leads to a negative acceleration and a decreasing velocity. All the phenomena agree well 
with the understanding of mechanics. Afterwards, the droplet slows down very quickly accompanying with an increasing distance from the left wedge end. Finally, the velocity will oscillate near zero, corresponding to a small oscillating distance from the left wedge end. The largest distance from the left wedge end is about $31.9 \mathrm{~nm}$ at this case and the maximum velocity of droplet is about $132.4 \mathrm{~m} / \mathrm{s}$, which is much faster than the achieved velocity of $1 \mathrm{~m} / \mathrm{s}$ on a wettability gradient surface using molecular dynamics simulations [18]. Similar comparable results are also reported in different experiments. The velocity of a droplet measured experimentally on a wedge-shaped surface is about $0.5 \mathrm{~m} / \mathrm{s}^{28}$, which is much higher than the experimental velocity $0.001 \mathrm{~m} / \mathrm{s}$ on a wettability gradient surface ${ }^{46}$. The present results directly verify the view of Khoo and Tseng ${ }^{28}$ that the design of a wedge-shaped functional surface may be of specific value to applications requiring higher moving velocity of droplets, such as fuel cells and semiconductors. Note that the maximum droplet velocity obtained in the MD simulations is actually much higher than that yielded experimentally ${ }^{28}$, which is due to two factors. (1) The substrate in MD simulations is always ideal atomically-smooth, which leads to a much smaller friction force, while the roughness and defects on a real surface are inevitable.

(2) The driving force per unit volume decreases as the droplet diameter increases as shown in Figure $4 \mathrm{~b}$ (details will be discussed later), leading to a larger driving force per unit volume acted on nano-droplets in MD simulations than that of a micro- or even macro-droplet in experiments.

During the motion, the instant configuration of droplets would change continuously due to the varying contact length between the boundary of water droplets and the boundary between hydrophobic and hydrophilic areas as shown in Figure 2c, where the configuration of a moving droplet is observed at different moments. It shows that the contact angle at each point on the 
contact line is not a constant at least until the droplet completely enters the hydrophilic wedge. At the initial stage, the droplet has a spherical shape. At the moment of $0.15 \mathrm{~ns}$, the droplet looks like a rugby, which is longer in the moving direction. As shown in the front view, both the trailing and leading sides of the droplet are in the hydrophilic region, the contact angle at the trailing side is about $55^{\circ}$ and the one at the leading side is $45^{\circ}$ as shown in Figure $2 \mathrm{c}$. Several reasons may induce different contact angles at the leading and trailing edges. (1) The shape of the droplet at $0.15 \mathrm{~ns}$ is not symmetric due to the wedge-shaped hydrophilic substrate surface, which is different from the case of a droplet on a symmetric surface. (2) The recorded contact angle is an instantaneous one since the droplet is moving. (3) It may be attributed to the less molecule number employed in the simulations, since the droplet adopted in the present simulation is relatively small. As a result, a strong fluctuation cannot be avoided. In the left view, the contact angle is about $100^{\circ}$ due to the effect of boundary of the hydrophobic and hydrophilic regions. As the droplet continues to move along the middle line, the droplet will totally enter the hydrophilic region. Finally, the droplet stops and achieves static equilibrium, the contact angle is about $51^{\circ}$ at all points on the contact line and the droplet becomes a spherical-cap.

\subsection{Theoretical model and analysis}

In order to disclose the mechanical mechanism, the potential energy of the water droplet during motion is shown in Figure 3a. It shows that the potential energy of the droplet decreases continuously as it moves forward and reaches to the minimum finally when the droplet stops in the hydrophilic zone. 

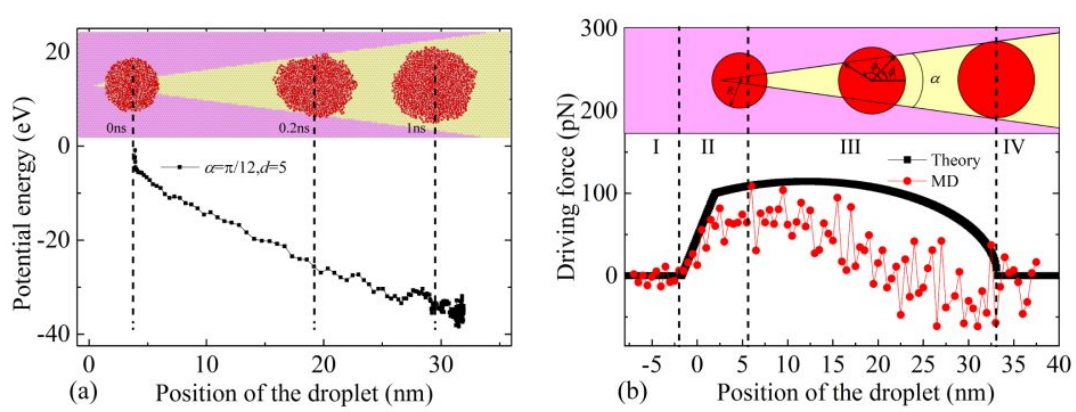

Figure 3. (a) Variation of the potential energy of water droplet during motion; (b) Comparison of the driving force on the water droplet predicted numerically and theoretically. The diameter of water droplet is $d 5 \mathrm{~nm}$ and the wedge angle $\alpha$ is $\pi / 12$.

A simple theoretical model can be adopted to further analyze the motion of droplet, in which both the driving force and friction force on the droplet are considered. The net force acted on the moving water droplet can be written as:

$$
F=F_{\mathrm{d}}-F_{\mathrm{f}}
$$

in which $F_{\mathrm{d}}$ and $F_{\mathrm{f}}$ denote the driving force and the friction force. For simplicity, it is assumed that the droplet is always spherical cap with a constant volume. The contact radius is assumed linearly increase as the droplet move from hydrophobic region to the hydrophilic one. Then, we have

$$
F_{\mathrm{d}}=\left\{\begin{array}{l}
0,\left(\mathrm{I}: x_{o}<-R_{2}\right) \\
2 R \gamma\left(\cos \theta_{1}-\cos \theta_{2}\right) \sin \phi_{1},\left(\mathrm{II}:-R_{2} \leq x_{0}<R_{0}\right) \\
2 R \gamma\left(\cos \theta_{1}-\cos \theta_{2}\right)\left(\sin \phi_{1}-\sin \phi_{2}\right),\left(\mathrm{III}: R_{0} \leq x_{0}<R_{1} / \sin (\alpha / 2)\right) \\
0,\left(\mathrm{IV}: x_{0} \geq R_{1} / \sin (\alpha / 2)\right)
\end{array}\right.
$$

and

$$
\begin{aligned}
& R=\frac{R_{1}-R_{2}}{R_{2} \sin (\alpha / 2)+R_{1}} \sin (\alpha / 2) x_{0}+\frac{R_{1} R_{2} \sin (\alpha / 2)+R_{1} R_{2}}{R_{2} \sin (\alpha / 2)+R_{1}} \\
& \phi_{1}=\arcsin \left(\frac{\sin (\alpha / 2)}{R} x_{0}\right)+\alpha / 2 \\
& \phi_{2}=\pi-\arcsin \left(\frac{\sin (\alpha / 2)}{R} x_{0}\right)+\alpha / 2
\end{aligned}
$$


where $\theta_{1}$ and $\theta_{2}$ are contact angles of water droplet on the hydrophilic and hydrophobic regions, respectively. $\gamma$ is the liquid-gas interfacial tension, $\alpha$ is the wedge angle. The detailed parameters are given in Table 1. $x_{0}$ is the $x$-coordinate with its origin at the center of the contact area. $\phi_{1}$ and $\phi_{2}$ represent azimuthal angles to the hydrophilic/hydrophobic boundary as shown in the inset of Figure $3 \mathrm{~b} . \mathrm{R}$ is the radius of the contact area, which linearly increases with $x_{0} . R_{1}$ and $R_{2}$ are the corresponding radii when the droplet is in hydrophilic and hydrophobic areas, respectively. $R_{0}$ is the radius when the trailing side lies at the wedge vertex.

Detailed expressions and analysis are given in Supporting Information.

Table 1: The parameter of the theoretical model

\begin{tabular}{cccc}
\hline$\theta_{1}\left({ }^{\circ}\right)$ & $\theta_{2}\left(^{\circ}\right)$ & $\gamma(\mathrm{mN} / \mathrm{m})$ & $\alpha$ \\
\hline $51^{\circ}$ & 138 & 72.8 & $\pi / 12$
\end{tabular}

According to the characteristic of driving force on the droplet, the whole moving process can be divided into 4 parts. For the I and IV part, the droplet is on hydrophobic and hydrophilic region, respectively, the corresponding driving forces are both zero. As for the II or III part, the droplet is across both hydrophobic and hydrophilic region, leading to a nonzero driving force. The contact radius $R$ and azimuthal angles $\phi_{1}$ and $\phi_{2}$ are function of $x$-coordinate of droplet center. Hence driving force varies with $x$-coordinate of droplet center. The whole variation tendency of the driving force is shown in Figure $3 b$.

According to the reference ${ }^{47}$, the friction force of water droplet across the wettability gradient interface can be written as:

$$
F_{\mathrm{f}}=-\left(g_{1} \lambda_{1} A_{1}+g_{2} \lambda_{2} A_{2}\right) v
$$


Where $v$ is the velocity of droplet. The subscripts 1 and 2 denote the hydrophilic and hydrophobic region, respectively. The parameters $g_{i}$ and $\lambda_{i}$ are geometry factors and friction coefficients of droplet on corresponding substrates, which are related to the wettability of substrates and can be found in reference ${ }^{47}$. The parameters $A_{i}$ are contact areas for droplet on substrates with different wettability.

From Eq. (4), it is found that the friction force is zero for a static droplet. However, the friction force would increase with the moving velocity of droplets. Once the friction force is larger than the driving force, the droplet would decelerate.

In order to arrive the driving force, we move the droplet on different positions along the middle line of the substrate and minimize its potential energy by fixing the center of mass of droplet. The force between the relaxed droplet and both hydrophilic and hydrophobic substrates is regarded as the driving force of droplet on the corresponding position. In this way, the driving force of droplet on any point along the middle line of substrate can be found. Both the driving force predicted theoretically and that achieved numerically as a function of its position are shown in Figure 3b, which can be divided into four parts according to its varying characteristics. Though the driving force varies nonlinearly, it is interesting to find that both results agree well with each other, at least for the overall trend. In part I, the driving force is zero since the whole droplet lies in the hydrophobic region, the droplet will never move. In part II, the leading side of the droplet starts to enter the hydrophilic region, the driving force increases almost linearly. When both the leading and trailing sides of the droplet enter the wedge-shaped hydrophilic area, the driving force would increase nonlinearly and then decrease nonlinearly until the entire droplet is 


\subsection{The effect of droplet size and wedge angle on droplet motion}

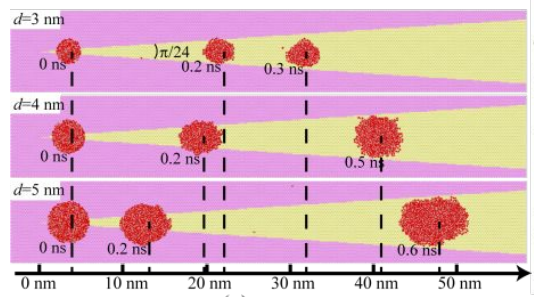

(a)

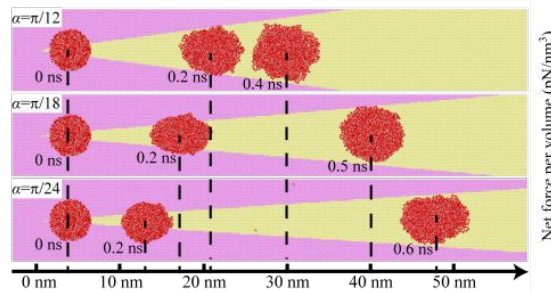

(d)

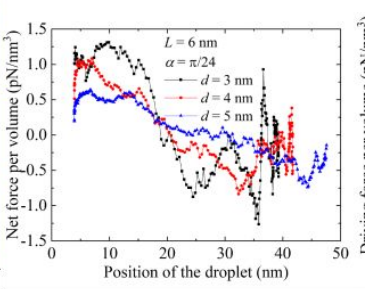

(b)

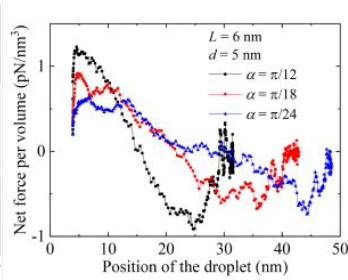

(e)

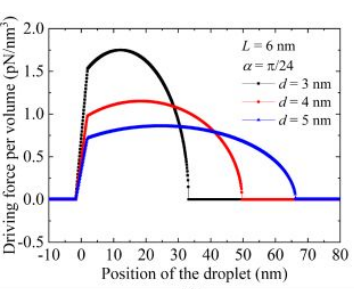

(c)

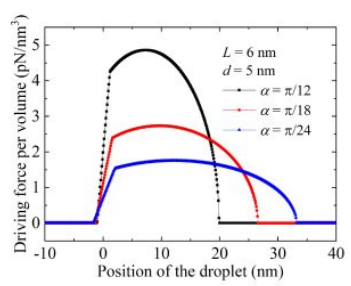

(f)

Figure 4. The effect of droplet diameter and wedge angle on the motion of droplet. (a) The snapshots, (b) net force and (c) the driving force per volume of water droplet for cases with droplets of different diameters $d=3$, 4 and $5 \mathrm{~nm}$ on the wedge-shaped functional surface, where the wedge angle is fixed as $\alpha=\pi / 24$. (d) The snapshots, (e) net force and (f) the driving force per volume of water droplet for cases with different wedge angles $\alpha=\pi / 12, \pi / 18$ and $\pi / 24$, but with a fixed droplet diameter $5 \mathrm{~nm}$.

The effect of droplet size and wedge angle of the hydrophilic zone on the moving behavior of droplets is further analyzed as shown in Figure 4. When the wedge angle is fixed as $\alpha=\pi / 24$, droplets of different diameters 3,4 and $5 \mathrm{~nm}$ are studied. We find that both the moving velocity 
and the final distance are influenced by the size of droplet. The smaller the droplet, the faster the initially moving velocity is. As shown in Figure $4 \mathrm{a}$, at the moment of $0.2 \mathrm{~ns}$, the smallest droplet moves the fastest. The maximum velocity of droplet decreases from $156.7 \mathrm{~m} / \mathrm{s}$ to $117.7 \mathrm{~m} / \mathrm{s}$ as the droplet diameter increases from $3 \mathrm{~nm}$ to $5 \mathrm{~nm}$. Such a result is consistent well with the experimental observation ${ }^{28}$. However, the smaller the droplet, the smaller the final distance will be. The droplets will stop at about $0.3,0.5$ and $0.6 \mathrm{~ns}$, respectively, for droplets of different diameters 3, 4 and $5 \mathrm{~nm}$ as shown in Figure 4a, which is because of the varying net force as shown in Figure $4 \mathrm{~b}$. The net force per volume of droplet at the initial stage increases as the droplet diameter decreases, leading to the largest velocity in the initial stage of the smallest droplet. As the droplet moves forward, the smaller the droplet, the faster the net force decreases, leading to a smaller moving distance for a smaller droplet. The driving force for droplets of different sizes is also given in Figure 4c for comparison.

For a droplet with a fixed diameter, we vary the wedge angle to check the moving behavior of the droplet as shown in Figure $4 d$, where the wedge angle $\alpha$ decreases from $\pi / 12$ to $\pi / 24$. It is found that with the smaller wedge angle, the initial moving velocity of droplet will be lower. Such a conclusion can be verified by the position of droplet at the moment of 0.2 ns as shown in Figure $4 \mathrm{~d}$. However, with the smaller wedge angle, the droplet will move farther as shown in Figure 4d, which can be explained by the net force acted on the droplet as shown in Figure 4e. The initial net force is the largest in the case with the largest wedge angle, leading to the fastest moving velocity of droplet on largest wedge angle. However, the net force will decrease faster in the case with a larger wedge angle, which leads to a shorter moving distance as shown in Figure 4d. The corresponding driving force is also given in Figure $4 \mathrm{f}$ for comparison. 


\section{RESULTS AND DISCUSSION}

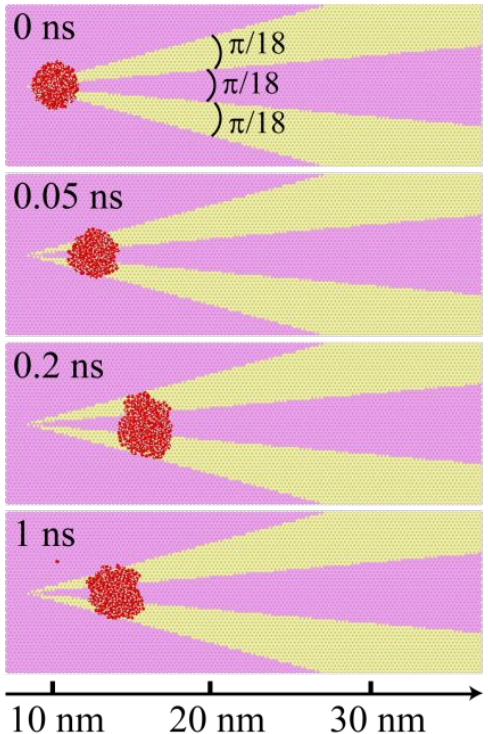

(a)

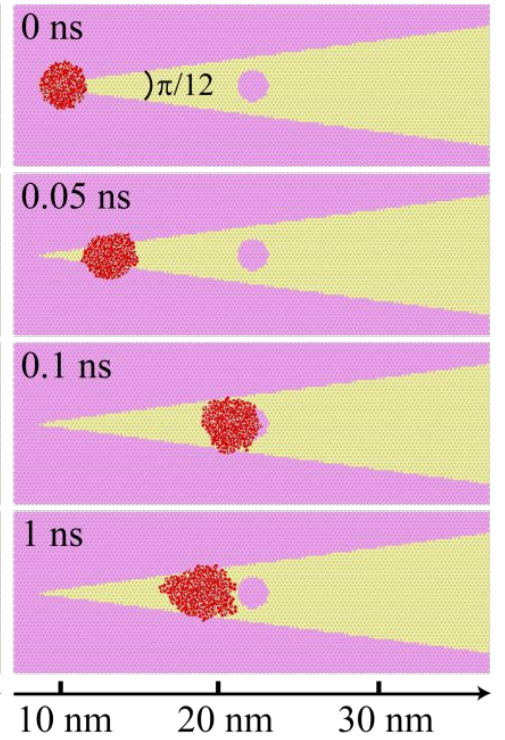

(b)

Figure 5. The pinning behavior of water droplet on different patterned surfaces. The droplet diameter is set to be $3 \mathrm{~nm}$. The yellow/pink indicates the hydrophilic/hydrophobic surface. (a) Multiple wedge-shaped functional strips owning the same angle $\pi / 18$ and sharing a common vertex. (b) A wedge-shaped hydrophilic strip with a wedge angle $\pi / 12$ decorated by a circular hydrophobic defect of diameter $2 \mathrm{~nm}$.

In order to control the stopping point of water droplets, several kinds of patterned surfaces are designed numerically as shown in Figure 5. A patterned surface containing three wedgeshaped functional strips with the same wedge angle $\pi / 18$ and a common vertex is shown in Figure 5a, where two hydrophilic strips are separated by a hydrophobic one. When a droplet locates at 
the vertex, it will move to the right due to the unbalanced force. Finally, it will stop and be pinned at a certain position, where the net force acted on the droplet should vanish. Numerical calculations demonstrate that the pinned position could be tuned flexibly by adjusting the wedge angle of the hydrophobic or hydrophilic area. The pinned position is farther whether a smaller hydrophilic wedge-shaped angle or a smaller hydrophobic wedge-shaped angle is adopted, for which typical examples can be found in Figures S7-8 of Supporting Information. Another simpler design is shown in Figure $5 \mathrm{~b}$ in order to pin a droplet with diameter $3 \mathrm{~nm}$. It is found that a circular hydrophobic defect at least $2 \mathrm{~nm}$ in diameter is needed in the hydrophilic region. Otherwise, the droplet may move over the defect. Detailed discussion can be found in Figure S4 of Supporting Information.

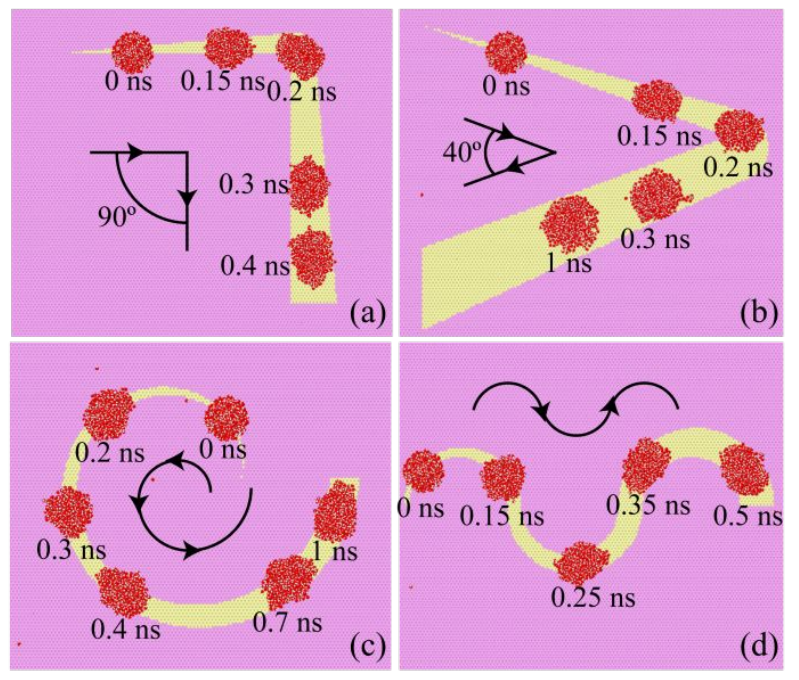

Figure 6. Nonlinear motion of a water droplet of diameter $3 \mathrm{~nm}$ on a patterned surface. The corner angles of the curve path are (a) $90^{\circ}$ and (b) $40^{\circ}$. The hydrophilic path is designed as (c) a spiral or (d) a sinusoid curve.

In order to control the moving direction of droplets, different curve paths could also be designed as the moving trajectory as shown in Figure 6. When a droplet is placed at the narrow end of the wedge-shaped curve path, it will move forward, turn 90 degrees, and keep going, as 
shown in Figure 6a. The wedge-shaped curve path with 40 degrees has been carefully studied. The droplet even placed near the corner can move over the corner for a droplet with diameter 3 $\mathrm{nm}$, as shown in Figure S5c, which may be due to the unbalanced force. The inertia of droplet does not play a role in this case. However, for a smaller droplet, $2.5 \mathrm{~nm}$ in diameter for example, the droplet placed near the corner will oscillate at the corner and does not move forward, which may be due to an approximately balanced force. Interestingly, if the droplet placed far from the corner, it can move over the corner as shown in Figures S5b,d. Therefore, it can be inferred that whether the droplet could move over the corner or not may depend on the coupling effect of its inertia and the net force. Furthermore, droplets can also be guided on a nonlinear path as shown in Figures $6 \mathrm{c}$ and $6 \mathrm{~d}$, it will move along the desired spiral or sinusoid curve path. Such a droplet path guidance should be of great value for droplet manipulations, which is highly expected in drug delivery and biomolecular motility ${ }^{48}$. The manipulation of droplets along a desirable nonlinear path is also presented $\mathrm{in}^{42}$, in which only a simple arc path was achieved based on the concept of wetting gradient. Actually, it is relatively difficult to fabricate a wettability gradient surface with complex trajectories $\mathrm{in}^{42}$, like a sinusoidal or spiral path. However, only two surfaces of different wettability are enough to realize the path control of droplets in the present way. 

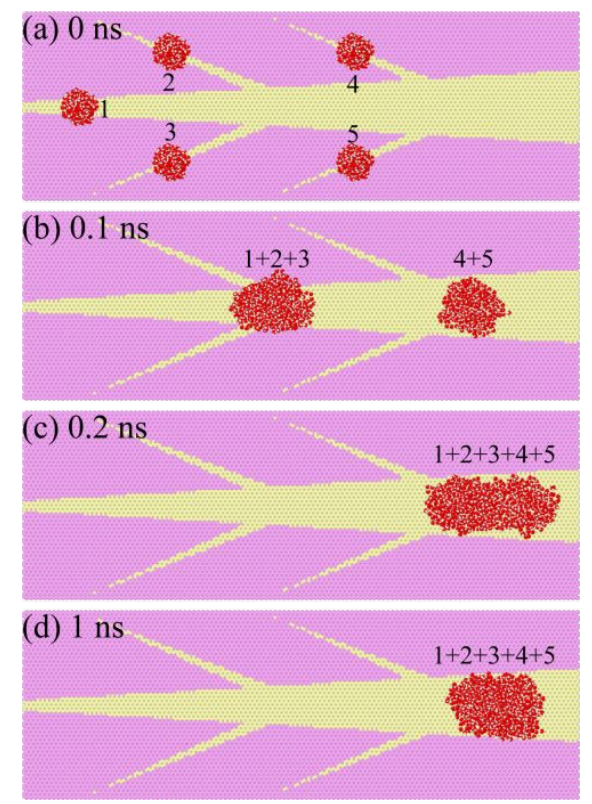

Figure 7. Snapshots of water aggregation on a patterned surface with multiple wedge-shaped tracks. The diameter of each water droplet is $2 \mathrm{~nm}$. The angles of the main track and lateral branches are $6.5^{\circ}$ and $3.4^{\circ}$, respectively.

Based on the above single wedge-shaped moving trajectory, more complicated system can be designed to realize an upgraded function of water aggregation as shown in Figure 7, where a root-like hydrophilic area can be designed on a hydrophobic surface. Small droplets of diameter $2 \mathrm{~nm}$ are placed at each end of four wedge-shaped lateral branches with wedge angle $3.4^{\circ}$, all of which are connected to a main trunk of wedge angle $6.5^{\circ}$. Each droplet is number as shown in Figure 7. It is found that droplets 1, 2 and 3 move toward the main trunk and aggregate from start to $0.1 \mathrm{~ns}$. Then, the aggregated droplet moves forward. Meantime, the same thing happens with droplets 4 and 5, which induces another aggregated droplet. The moving velocity of the aggregated droplet forming by droplets 1, 2 and 3 is faster than that forming by droplets 4 and 5, consistent with the result in the above single wedge-shaped case. Finally, two aggregated droplets combine into a larger one at $0.2 \mathrm{~ns}$ as shown in Figure 7c, which moves forward and stops at a certain position. Typical movie can be found in S2 of Supporting Information. Such a multi-track wedge-shaped surface has been successfully reported recently in experiments ${ }^{30}$. All 
the numerical results agree well with the experimental observations. Based on such an interesting phenomenon, systematic study is feasible, including the effect of lateral branch angle, symmetrical feature of lateral branches, existence of defects, etc. For reasons of length, interesting results in this area will be presented systematically in the near future.

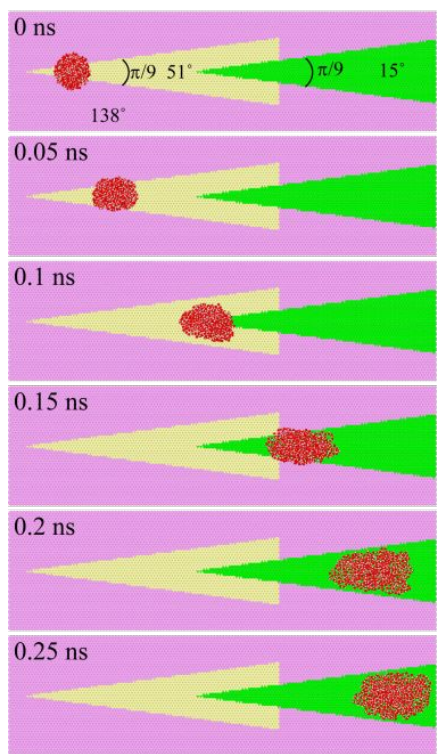

(a)

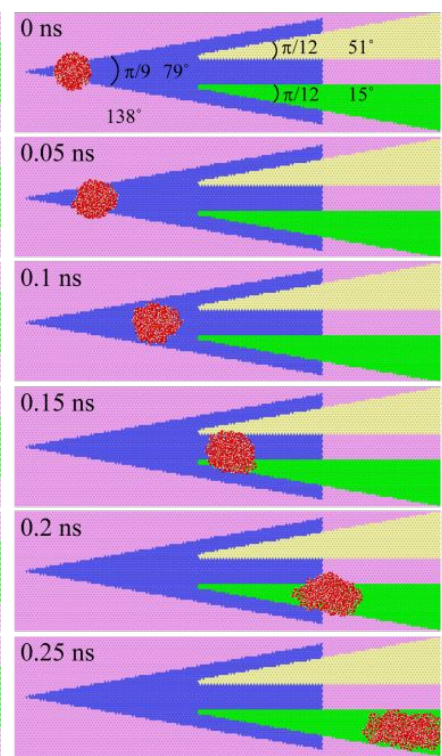

(b)

Figure 8. Snapshots of water acceleration and path selection on a patterned surface with multiple wedge-shaped tracks. The diameter of water droplets is set to be $3 \mathrm{~nm}$. (a) Contact angles of the pink, yellow and green areas are $138^{\circ}, 51^{\circ}$ and $15^{\circ}$, respectively. (b) Contact angles of the pink, blue, yellow and green are $138^{\circ}, 79^{\circ}, 51^{\circ}$ and $15^{\circ}$, respectively.

The moving velocity and the transport distance of droplets can also be improved by the combination of simple wedge-shaped surfaces with different wettability. Different designs can be simply realized as shown in Figure 8 . The pink hydrophobic surface has a contact angle of $138^{\circ}$, while the yellow and green hydrophilic wedge-shaped parts have contact angles of $51^{\circ}$ and $15^{\circ}$, respectively, as shown in Figure $8 \mathrm{a}$. When a droplet of diameter $3 \mathrm{~nm}$ is placed at the end of the yellow wedge-shaped part, it will move forward. If the green hydrophilic wedge-shaped part is well designed, the droplet could get to the end of green part. Subsequently, the droplet will enter the green wedge-shaped area and move forward. Not only the transport distance but 
also the effective moving velocity can be significantly improved. Furthermore, if the functional surface is well designed as that shown in Figure 8b, where contact angles of the pink, blue, yellow and green areas are $138^{\circ}, 79^{\circ}, 51^{\circ}$ and $15^{\circ}$, respectively, a water droplet could be transported from the end of the blue wedge-shaped area to the more hydrophilic green area with a contact angle of $15^{\circ}$ to realize path selection. Such a design may be useful for the separation of droplets of different wettability.

In fact, it is feasible to fabricate the above design of functional surfaces. The wettability of a solid surface can be modified effectively by the surface chemical composition ${ }^{17}$ and microstructures ${ }^{18-19}$. With these methods, patterned surfaces with different wettability could be achieved. A pillar-structured surface with gradient pillar sizes was designed to drive $\operatorname{droplet}^{19}$, the stripe-patterned gradient surface was created via fluorinated self-assembled mono-layers and $\mathrm{SiO}_{2}$ substrate to control droplet motion ${ }^{26}$. In addition, the surface wettability could also be modified by external fields, for example, a reversibly switching surface could be design in response to electrical potential by controlling the molecular conformations ${ }^{20}$.

\section{CONCLUSIONS}

Using MD simulations and theoretical analysis, we present the novel idea that includes the study of driving mechanism of water droplet moving on a wedge-shaped functional surface and is capable of designing complex manipulation methods for droplet motion. Spontaneous droplet motion on hydrophilic wedge-shape surface by inset on hydrophobic surface are achieved. Along with the moving velocity, we can also tune the transport distance by changing the wedge angle and water droplet size. Based on the wedge-shaped transport technique, several novel designs of functional surfaces are proposed above, through which complicated manipulations can be 
achieved. The transport distance of droplets can be controlled precisely through the pinning behavior on a multiple wedge-shaped surface or wedge-shaped surface with hydrophobic defect. The transport direction can also be controlled by the area-gradient hydrophilic surface, even with a sharp turning angle, spiral or sinusoidal curve path. Combination of multiple wedge-shaped functional paths, the droplet aggregation will be achieved finally. Furthermore, the moving velocity and transport distance could be improved through multiple tracks with different wettability, based on which path selection can be achieved simultaneously. The results and the novel technique presented in this paper can be very useful for the design of functional surfaces to control and manipulate droplet motion, even also for separation of droplets of different wettability. This work may lead foundation for further exploration in this area of research. 


\title{
AUTHOR INFORMATION \\ Corresponding Author \\ *E-mail: wangchao@lnm.imech.ac.cn (C.W). \\ *E-mail: chenshaohua72@hotmail.com (S.H.C). \\ Notes \\ The authors declare no competing financial interest.
}

\section{ACKNOWLEDGEMENTS}

The work reported here is supported by NSFC through Grants \#11532013, \#11872114, \#11602270.

MD simulations were carried out at Supercomputing Center of Lv Liang Cloud Computing Center in China.

\author{
ASSOCIATED CONTENT \\ Supporting Information \\ The Supporting Information is available free of charge via the Internet at http://pubs.acs.org. \\ Further details about the theoretical analysis and the moving behavior (PDF). Typical \\ movie about the droplet motion on a wedge-shaped functional surface (S1) and water \\ aggregation on a multi-track wedge-shaped surface (S2).
}




\section{References}

1. Wang, C.; Chen, S. H., Motion Driven by Strain Gradient Fields. Sci. Rep. 2015, 5, 13675.

2. Chang, T. C.; Zhang, H. W.; Guo, Z. R.; Guo, X. M.; Gao, H. J., Nanoscale Directional Motion Towards Regions of Stiffness. Phys. Rev. Lett. 2015, 114, 015504.

3. Dai, C. C.; Guo, Z. R.; Zhang, H. W.; Chang, T. C., Nanoscale Linear-to-Linear Motion Converter of Graphene. Nanoscale 2016, 8, 14406.

4. Wang, S.; Wang, C.; Peng, Z.; Chen, S., A New Technique for Nanoparticle Transport and Its Application in a Novel Nano-Sieve. Sci. Rep. 2018, 8, 9682.

5. Becton, M.; Wang, X., Thermal Gradients on Graphene to Erive Nanoflake Motion. J. Chem. Theory Comput. 2014, 10, 722-30.

6. Sas, I.; Gorga, R. E.; Joines, J. A.; Thoney, K. A., Literature Review on Superhydrophobic Self - Cleaning Surfaces Produced by Electrospinning. J. Polym. Sci. Pol. Phys. 2012, 50, 824845.

7. Blossey, R., Self-Cleaning Surfaces-Virtual Realities. Nat. Mater. 2003, 2, 301-306.

8. Liu, H.; Szunerits, S.; Xu, W.; Boukherroub, R., Preparation of Superhydrophobic Coatings on Zinc as Effective Corrosion Barriers. ACS Appl. Mater. Inter. 2009, 1, 1150-1153.

9. Cao, L.; Jones, A. K.; Sikka, V. K.; Wu, J.; Gao, D., Anti-Icing Superhydrophobic Coatings. Langmuir 2009, 25, 12444-12448.

10. Parker, A. R.; Lawrence, C. R., Water Capture by a Desert Beetle. Nature 2001, 414, 33-34. 11. Garrod, R. P.; Harris, L. G.; Schofield, W. C.; Mcgettrick, J.; Ward, L. J.; Teare, D. O.; Badyal, J. P., Mimicking a Stenocara Beetle's Back for Microcondensation Using Plasmachemical Patterned Superhydrophobic-Superhydrophilic Surfaces. Langmuir 2007, 23, 689-693.

12. Chaudhury, M. K.; Whitesides, G. M., How to Make Water Run Uphill. Science 1992, 256, 1539-1541.

13. Huang, D.-J.; Leu, T.-S., Fabrication of a Wettability-Gradient Surface on Copper by Screen-Printing Techniques. J. Micromech. Microeng. 2015, 25, 085007.

14. Teh, S. Y.; Lin, R.; Hung, L. H.; Lee, A. P., Droplet Microfluidics. Lab. Chip 2008, 8, 198220.

15. Gondal, M. A.; Sadullah, M. S.; Dastageer, M. A.; McKinley, G. H.; Panchanathan, D.; Varanasi, K. K., Study of Factors Governing Oil-Water Separation Process Using Tio2 Films Prepared by Spray Deposition of Nanoparticle Dispersions. ACS Appl. Mater. Inter. 2014, 6, 13422-13429.

16. Lu, G.; Wang, X.-D.; Duan, Y.-Y., A Critical Review of Dynamic Wetting by Complex Fluids: From Newtonian Fluids to Non-Newtonian Fluids and Nanofluids. Adv. colloid interface Sci. 2016, 236, 43-62.

17. Xue, C.-H.; Jia, S.-T.; Chen, H.-Z.; Wang, M., Superhydrophobic Cotton Fabrics Prepared by Sol-Gel Coating of Tio2 and Surface Hydrophobization. Sci. Technol. Adv. Mat. 2008, 9, 035001 .

18. Yoshimitsu, Z.; Nakajima, A.; Watanabe, T.; Hashimoto, K., Effects of Surface Structure on the Hydrophobicity and Sliding Behavior of Water Droplets. Langmuir 2002, 18, 5818-5822. 19. Zheng, Q. S.; Lv, C. J.; Hao, P. F.; Sheridan, J., Small Is Beautiful, and Dry. Sci. China- 
Phys. Mech. Astron. 2010, 53, 2245-2259.

20. Lahann, J.; Mitragotri, S.; Tran, T.-N.; Kaido, H.; Sundaram, J.; Choi, I. S.; Hoffer, S.; Somorjai, G. A.; Langer, R., A Reversibly Switching Surface. Science 2003, 299, 371-374.

21. Wang, R.; Hashimoto, K.; Fujishima, A.; Chikuni, M.; Kojima, E.; Kitamura, A.; Shimohigoshi, M.; Watanabe, T., Light-Induced Amphiphilic Surfaces. Nature 1997, 388, 431. 22. Zhang, J.; Li, J.; Han, Y., Superhydrophobic Ptfe Surfaces by Extension. Macromol. Rapid Comm. 2004, 25, 1105-1108.

23. Shirtcliffe, N. J.; Mchale, G.; Newton, M. I.; Perry, C. C.; Roach, P., Porous Materials Show Superhydrophobic to Superhydrophilic Switching. Chem. Commun. 2005, 25, 3135-3137.

24. Halverson, J. D.; Maldarelli, C.; Couzis, A.; Koplik, J., A Molecular Dynamics Study of the Motion of a Nanodroplet of Pure Liquid on a Wetting Gradient. J. Chem. Phys. 2008, 129, 164708 .

25. Subramanian, R. S.; Moumen, N.; McLaughlin, J. B., Motion of a Drop on a Solid Surface Due to a Wettability Gradient. Langmuir 2005, 21, 11844-11849.

26. Bliznyuk, O.; Jansen, H. P.; Kooij, E. S.; Zandvliet, H. J.; Poelsema, B., Smart Design of Stripe-Patterned Gradient Surfaces to Control Droplet Motion. Langmuir 2011, 27, 11238 11245.

27. Zhang, J.; Han, Y., Shape-Gradient Composite Surfaces: Water Droplets Move Uphill. Langmuir 2007, 23, 6136-6141.

28. Khoo, H. S.; Tseng, F.-G., Spontaneous High-Speed Transport of Subnanoliter Water Droplet on Gradient Nanotextured Surfaces. Appl. Phys. Lett. 2009, 95, 063108.

29. Alheshibri, M.; Rogers, N.; Sommers, A.; Eid, K., Spontaneous Movement of Water Droplets on Patterned Cu and Al Surfaces with Wedge-Shaped Gradients. Appl. Phys. Lett. 2013, 102, 174103.

30. Zheng, Y.; Cheng, J.; Zhou, C.; Xing, H.; Wen, X.; Pi, P.; Xu, S., Droplet Motion on a Shape Gradient Surface. Langmuir 2017, 33, 4172-4177.

31. Ghosh, A.; Ganguly, R.; Schutzius, T. M.; Megaridis, C. M., Wettability Patterning for High-Rate, Pumpless Fluid Transport on Open, Non-Planar Microfluidic Platforms. Lab. Chip 2014, 14, 1538-1550.

32. Ody, T.; Panth, M.; Sommers, A.; Eid, K., Controlling the Motion of Ferrofluid Droplets Using Surface Tension Gradients and Magnetoviscous Pinning. Langmuir 2016, 32, 6967-6976.

33. Atencia, J.; Beebe, D. J., Controlled Microfluidic Interfaces. Nature 2005, 437, 648-55.

34. González, M. A.; Abascal, J. L., The Shear Viscosity of Rigid Water Models. J. Chem. Phys. 2010, 132, 096101.

35. Berendsen, H. J. C.; Grigera, J. R.; Straatsma, T. P., The Missing Term in Effective Pair Potentials. J. Phys. Chem. 1987, 91, 6269-6271.

36. Ryckaert, J. P.; Ciccotti, G.; Berendsen, H. J. C., Numerical Integration of the Cartesian Equations of Motion of a System with Constraints: Molecular Dynamics of N-Alkanes. $J$. Comput. Phys. 1977, 23, 327-341.

37. Jorgensen, W. L.; Chandrasekhar, J.; Madura, J. D.; Impey, R. W.; Klein, M. L., Comparison of Simple Potential Functions for Simulating Liquid Water. J. Chem. Phys. 1983, 79, 926-935.

38. Zielkiewicz, J., Structural Properties of Water: Comparison of the Spc, Spce, Tip4p, and Tip5p Models of Water. J. Chem. Phys. 2005, 123, 104501.

39. Hockney, R. W.; Eastwood, J. W., Computer Simulation Using Particles; crc Press, 1988. 
40. Stuart, S. J.; Tutein, A. B.; Harrison, J. A., A Reactive Potential for Hydrocarbons with Intermolecular Interactions. J. Chem. Phys. 2000, 112, 6472-6486.

41. Lv, C. J.; Chen, C.; Chuang, Y.-C.; Tseng, F.-G.; Yin, Y. J.; Grey, F.; Zheng, Q. S., Substrate Curvature Gradient Drives Rapid Droplet Motion. Phys. Rev. Lett. 2014, 113, 026101.

42. Liu, Q.; Xu, B., Actuating Water Droplets on Graphene Via Surface Wettability Gradients. Langmuir 2015, 31, 9070-9075.

43. Ashraf, A.; Wu, Y.; Wang, M. C.; Yong, K.; Sun, T.; Jing, Y.; Haasch, R. T.; Aluru, N. R.; Nam, S., Doping-Induced Tunable Wettability and Adhesion of Graphene. Nano lett. 2016, 16, 4708-4712.

44. Wei, N.; Lv, C.; Xu, Z., Wetting of Graphene Oxide: A Molecular Dynamics Study. Langmuir 2014, 30, 3572-2578.

45. Plimpton, S., Fast Parallel Algorithms for Short-Range Molecular Dynamics. J. Comput. Phys. 1993, 117, 1-19.

46. Daniel, S.; Chaudhury, M. K., Rectified Motion of Liquid Drops on Gradient Surfaces Induced by Vibration. Langmuir 2002, 18, 3404-3407.

47. Liu, Q.; Xu, B., A Unified Mechanics Model of Wettability Gradient-Driven Motion of Water Droplet on Solid Surfaces. Extreme Mech. Lett. 2016, 9, 304-309.

48. Zhang, J.; Yao, Y.; Sheng, L.; Liu, J., Self-Fueled Biomimetic Liquid Metal Mollusk. Adv. Mater. 2015, 27, 2648. 

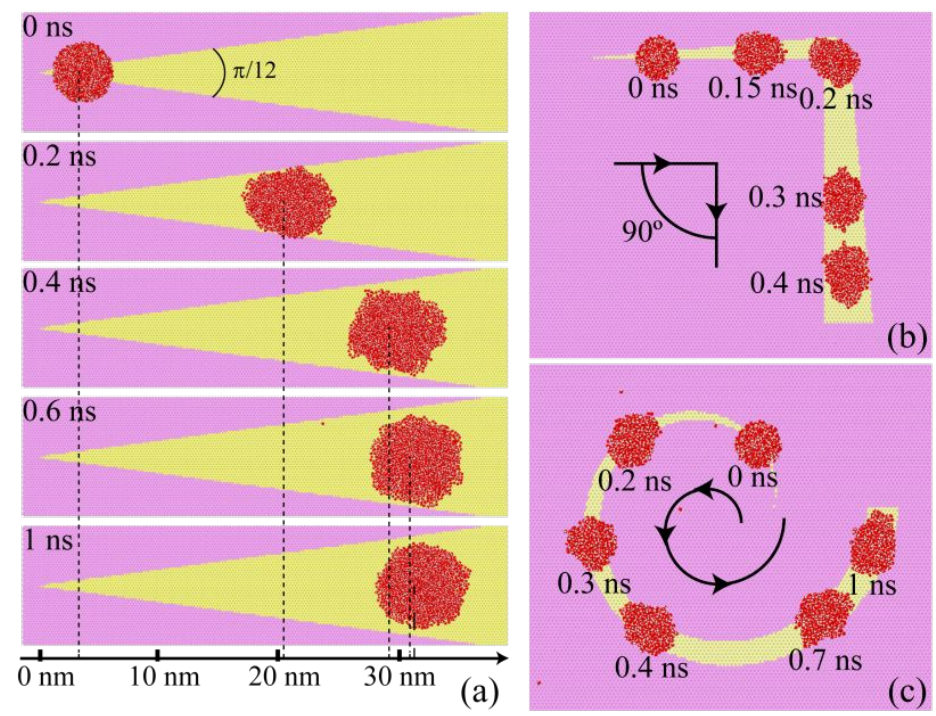

TOC Graphic 


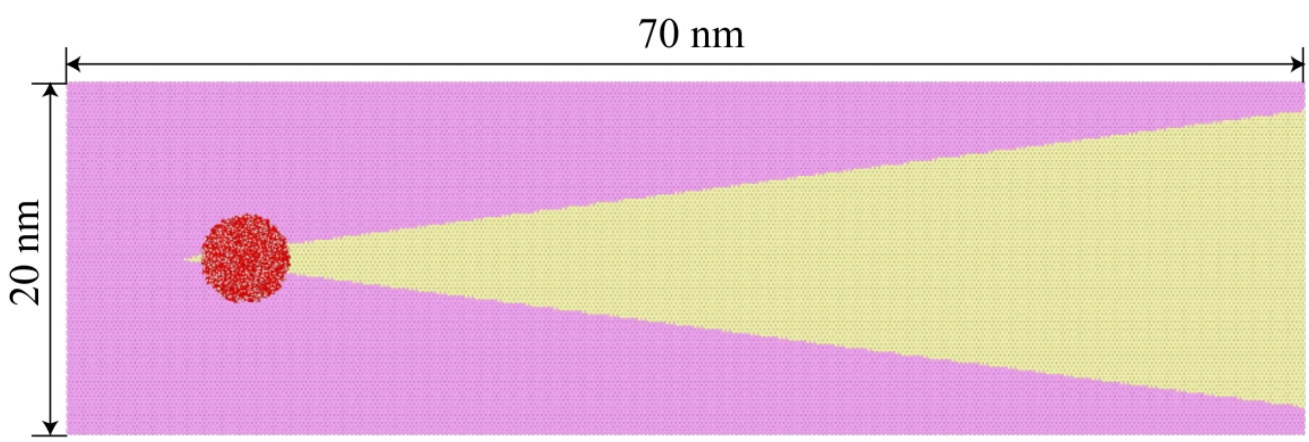

(a) top view

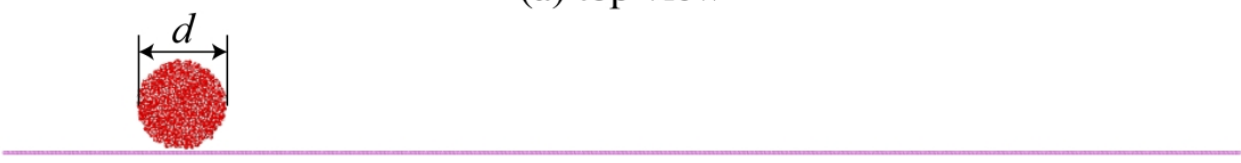

(b) front view

Figure 1. Schematic of the simulation system. (a) the top view; (b) the front view. $259 \times 134 \mathrm{~mm}(300 \times 300 \mathrm{DPI})$ 
(a) (b)

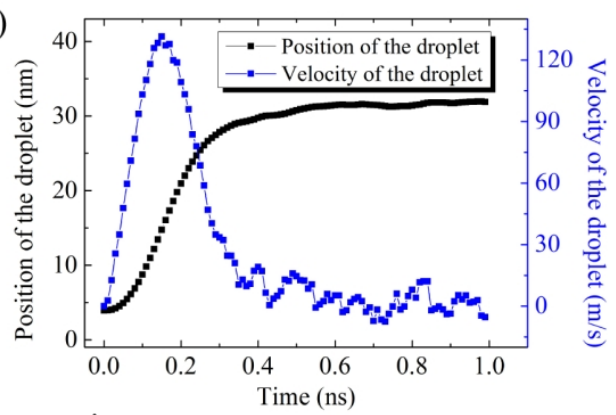

(c) top view
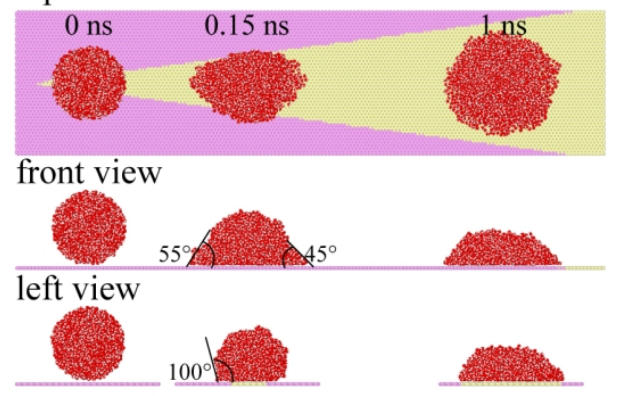

Figure 2. A water droplet moves spontaneously within an embedded wedge-shaped region on a graphene sheet. (a) Snapshots of the position of droplet on the wedge-shaped functional surface at $0,0.2,0.4,0.6$ and $1 \mathrm{~ns}$; (b) Position and velocity of the water droplet as a function of the collapsed time; (c) Snapshots of the configuration of a moving droplet observed in different directions. The diameter of the droplet $d$ is $5 \mathrm{~nm}$. 

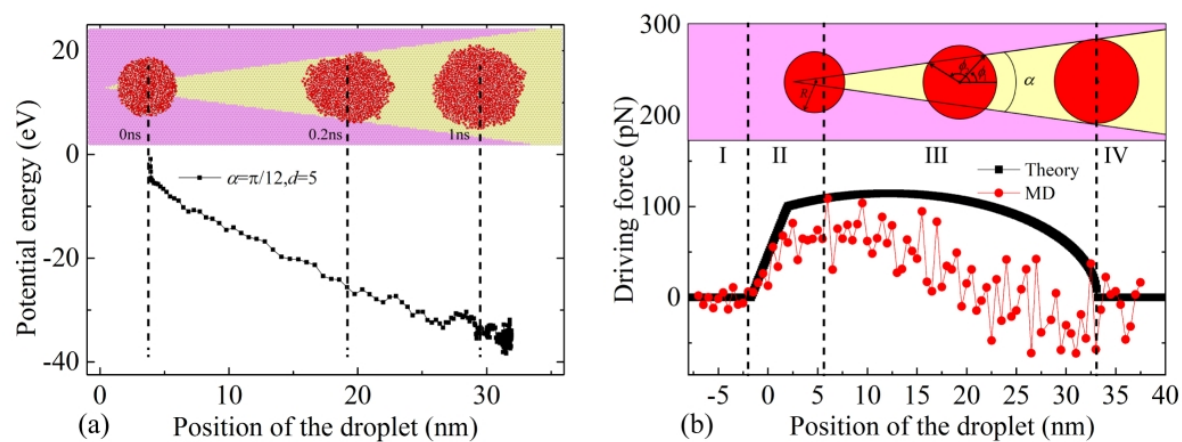

Figure 3. (a) Variation of the potential energy of water droplet during motion; (b) Comparison of the driving force on the water droplet predicted numerically and theoretically. The diameter of water droplet $d$ is $5 \mathrm{~nm}$ and the wedge angle $a$ is $n / 12$. 


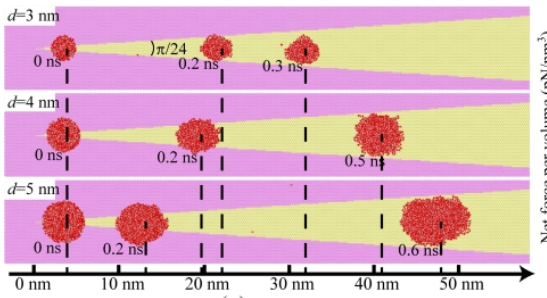

(a)

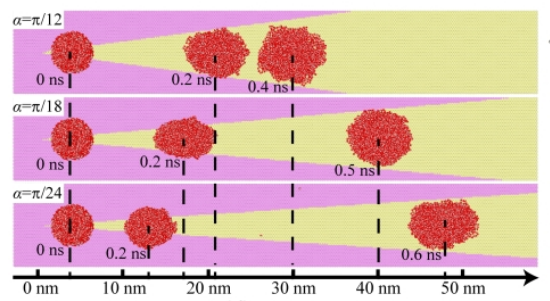

(d)

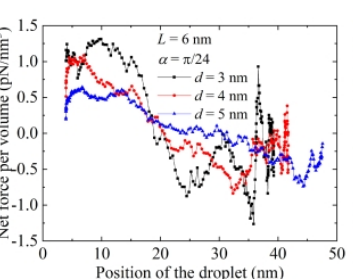

(b)

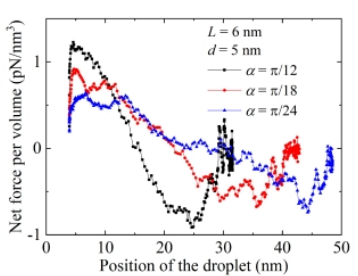

(e)

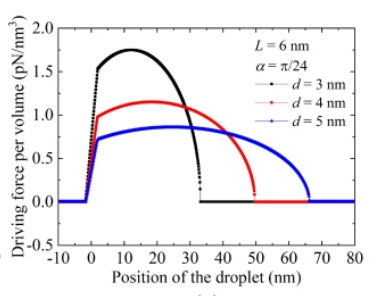

(c)

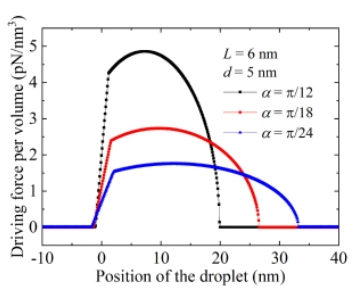

(f)

Figure 4. The effect of droplet diameter and wedge angle on the motion of droplet. (a) The snapshots, (b) net force and (c) the driving force per volume of water droplet for cases with droplets of different diameters $d=3,4$ and $5 \mathrm{~nm}$ on the wedge-shaped functional surface, where the wedge angle is fixed as $a=n / 24$. (d) The snapshots, (e) net force and (f) the driving force per volume of water droplet for cases with different wedge angles $a=n / 12, \Pi / 18$ and $n / 24$, but with a fixed droplet diameter $5 \mathrm{~nm}$. 

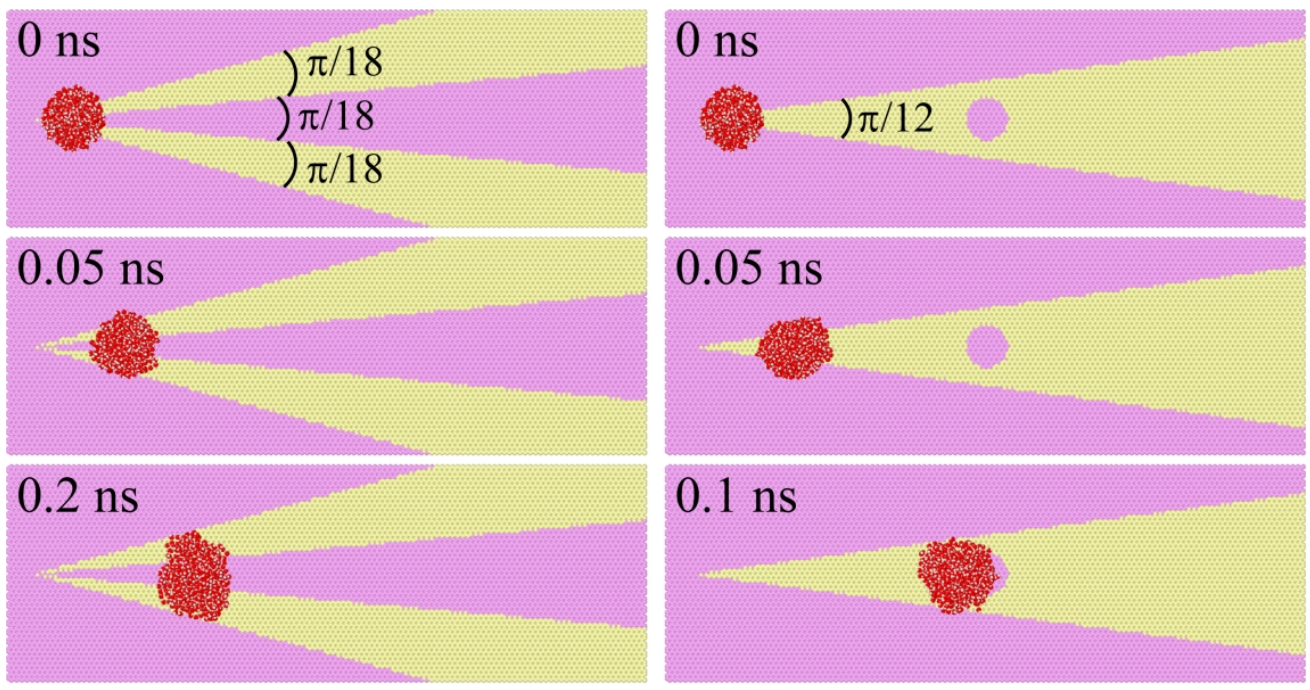

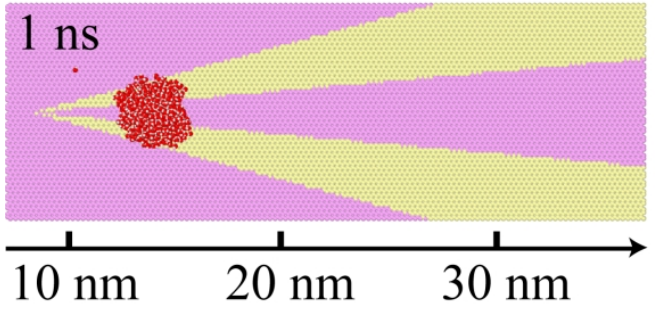

(a)

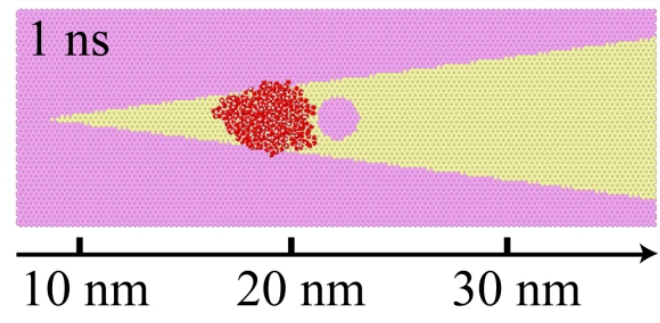

(b)

Figure 5. The pinning behavior of water droplet on different patterned surfaces. The droplet diameter is set to be $3 \mathrm{~nm}$. The yellow/pink indicates the hydrophilic/hydrophobic surface. (a) Multiple wedge-shaped functional strips owning the same angle $n / 18$ and sharing a common vertex. (b) A wedge-shaped hydrophilic strip with a wedge angle $n / 12$ decorated by a circular hydrophobic defect of diameter $2 \mathrm{~nm}$.

$$
214 \times 174 \mathrm{~mm}(300 \times 300 \mathrm{DPI})
$$



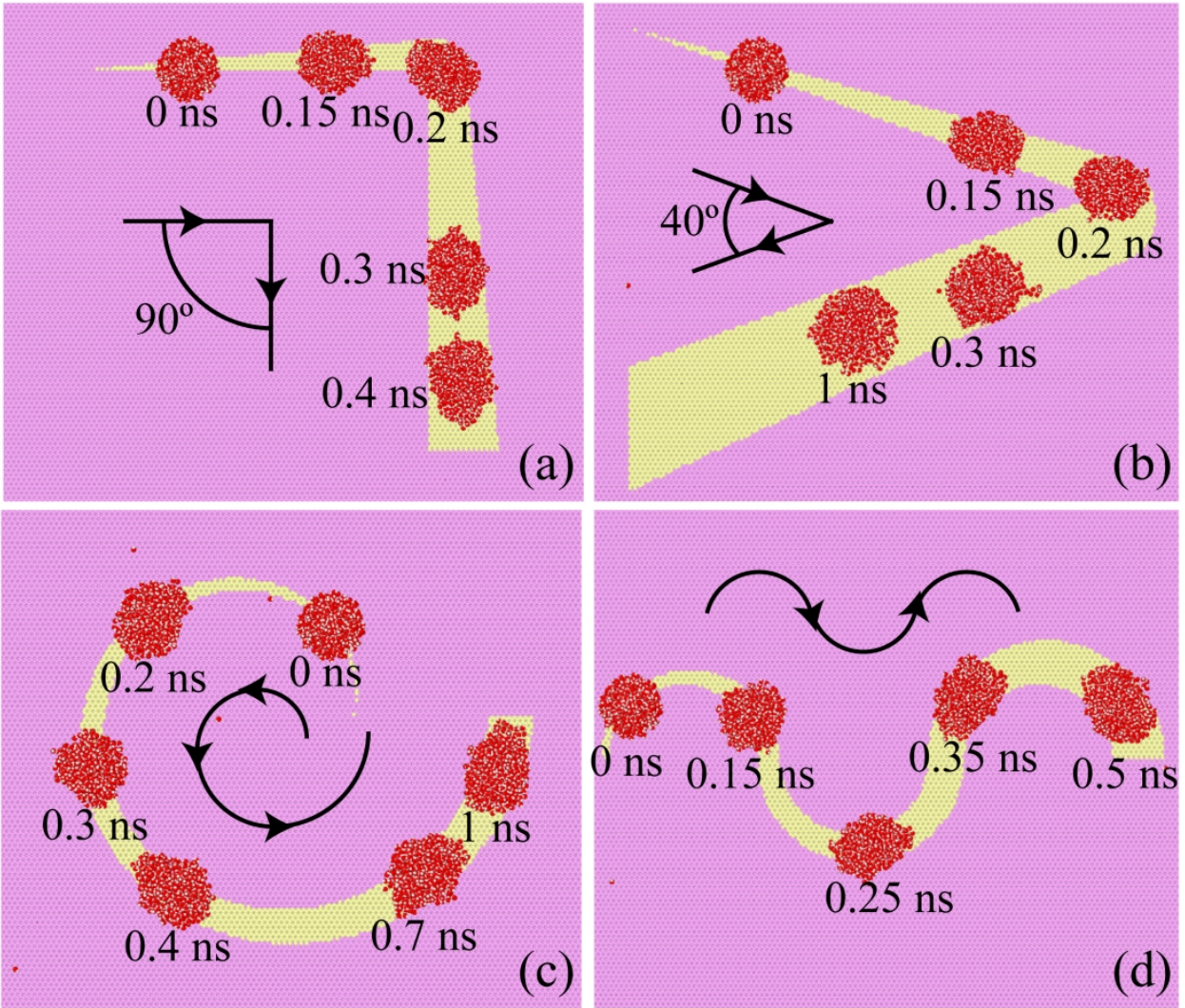

Figure 6. Nonlinear motion of a water droplet of diameter $3 \mathrm{~nm}$ on a patterned surface. The corner angles of the curve path are (a) $90^{\circ}$ and (b) $40^{\circ}$. The hydrophilic path is designed as (c) a spiral or (d) a sinusoid curve.

$204 \times 197 \mathrm{~mm}(300 \times 300$ DPI) 

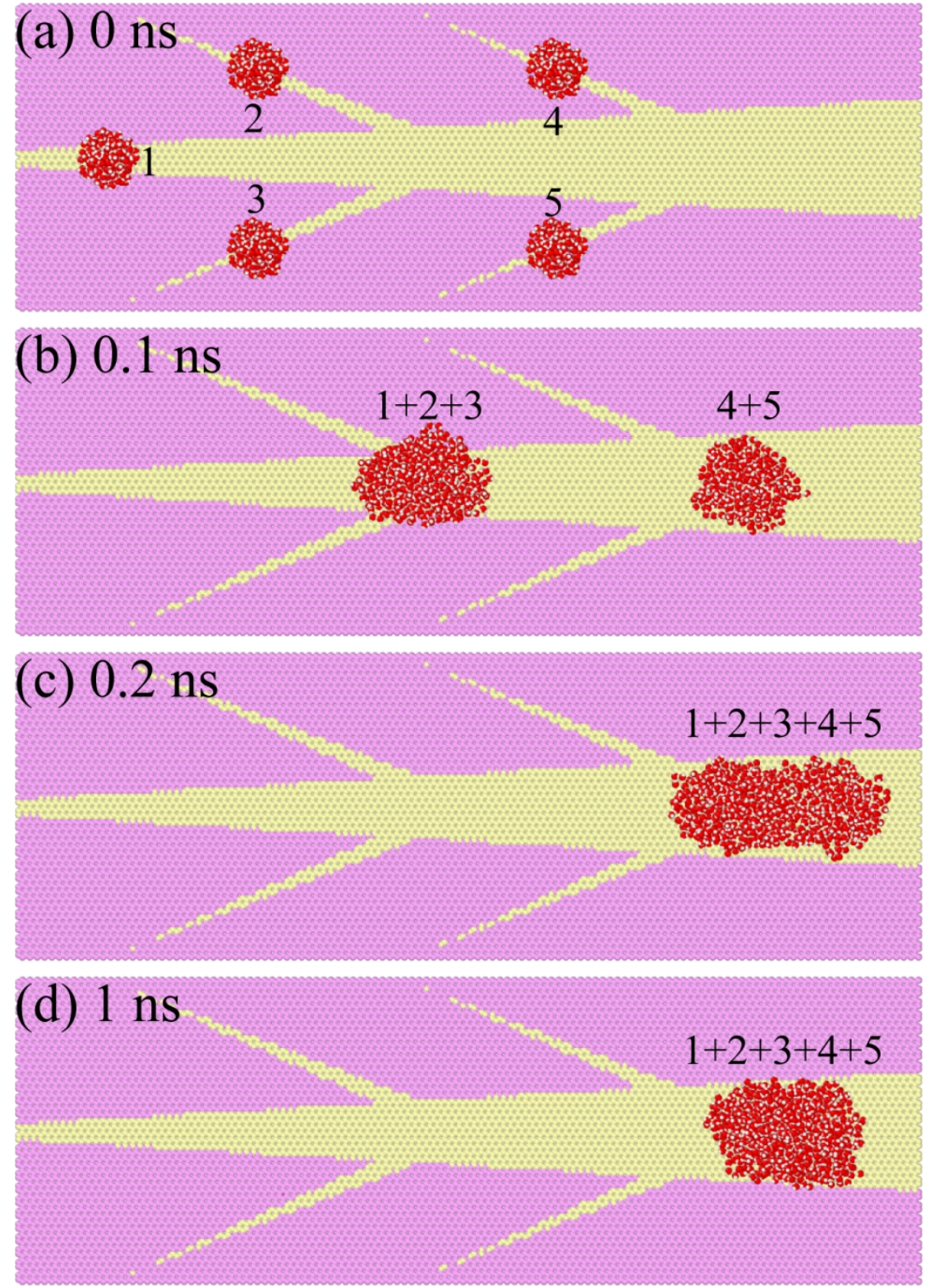

Figure 7. Snapshots of water aggregation on a patterned surface with multiple wedge-shaped tracks. The diameter of each water droplet is $2 \mathrm{~nm}$. The angles of the main track and lateral branches are $6.5^{\circ}$ and $3.4^{\circ}$, respectively.

$105 \times 149 \mathrm{~mm}(300 \times 300 \mathrm{DPI})$ 

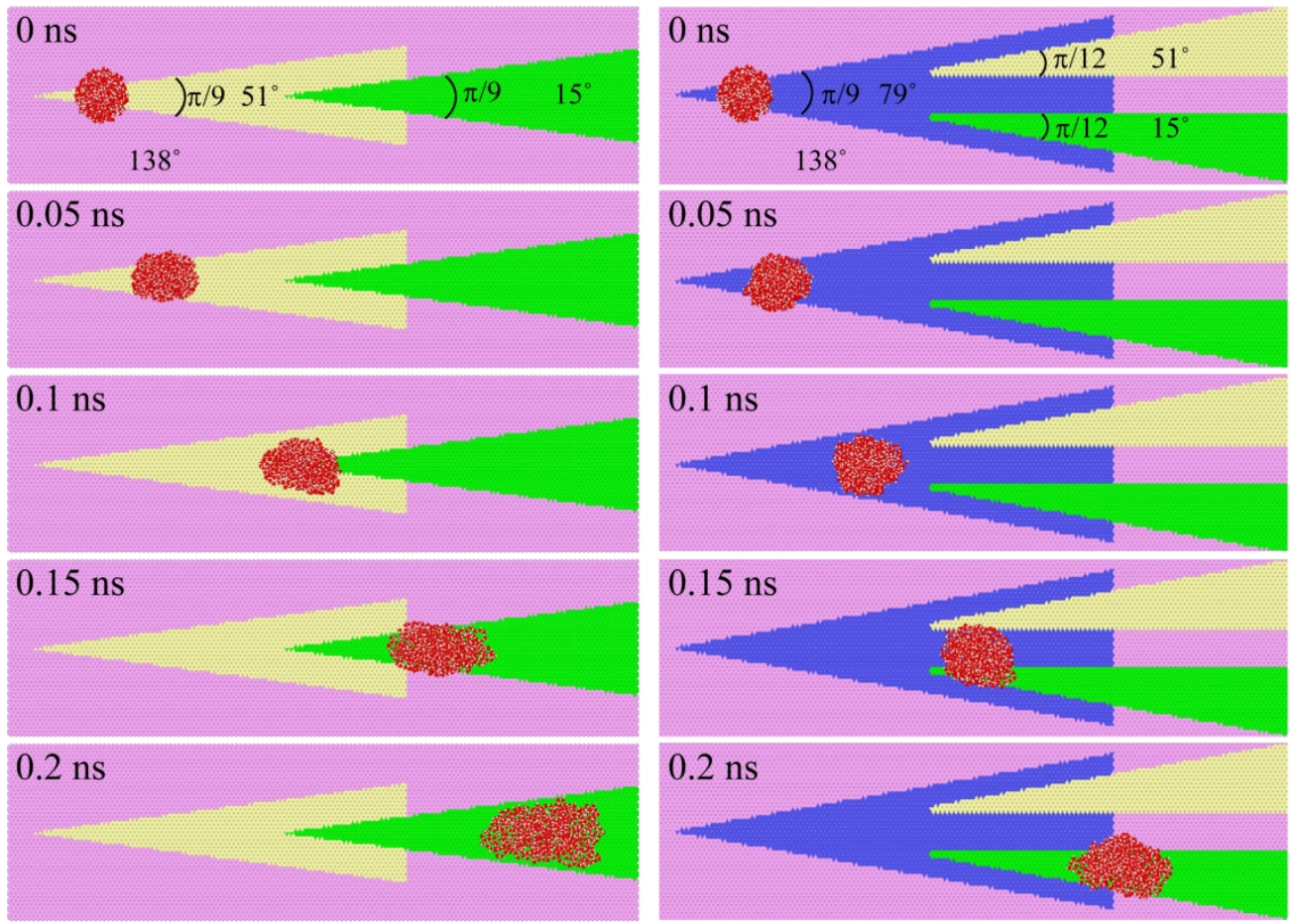

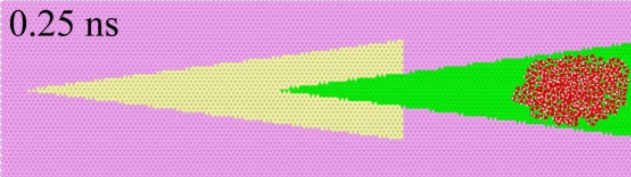

(a)

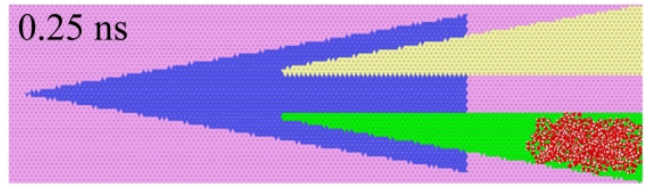

(b)

Figure 8: Snapshots of water acceleration and path selection on a patterned surface with multiple wedgeshaped tracks. The diameter of water droplets is set to be $3 \mathrm{~nm}$. (a) Contact angles of the pink, yellow and green areas are $138^{\circ}, 51^{\circ}$ and $15^{\circ}$, respectively. (b) Contact angles of the pink, blue, yellow and green are $138^{\circ}, 79^{\circ}, 51^{\circ}$ and $15^{\circ}$, respectively.

$216 \times 194 \mathrm{~mm}(300 \times 300 \mathrm{DPI})$ 

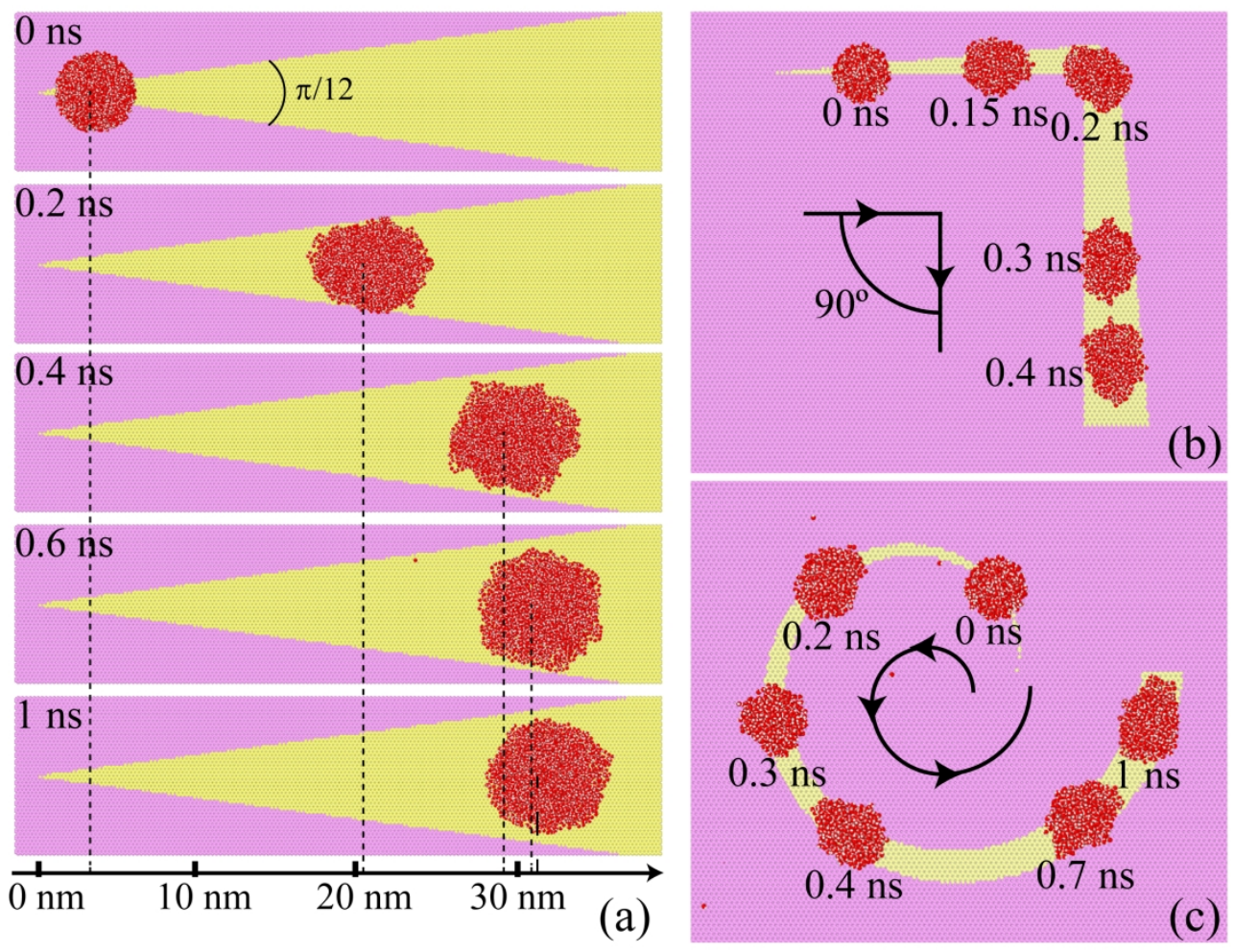

TOC Graphic

$222 \times 187 \mathrm{~mm}(300 \times 300$ DPI) 